\title{
Liftable vector fields, unfoldings and augmentations
}

\section{J. J. Nuño-Ballesteros ${ }^{1} \cdot$ R. Oset Sinha $^{1}$ (D)}

Received: 16 January 2019 / Accepted: 30 July 2021 / Published online: 7 August 2021

(c) The Author(s) 2021

\begin{abstract}
We study liftable vector fields of smooth map-germs. We show how to obtain the module of liftable vector fields of any map-germ of finite singularity type from the module of liftable vector fields of a stable unfolding of it. As an application, we obtain the liftable vector fields for the family $H_{k}$ in Mond's list. We then show the relation between the liftable vector fields of a stable germ and its augmentations.
\end{abstract}

Keywords Liftable vector fields · Augmentations $\cdot$ Stable unfoldings

Mathematics Subject Classification Primary 58K40; Secondary 58K20 · 32S05

\section{Introduction}

Since the beginning of the study of singularities of differentiable maps with Whitney and Thom, classification of singularities has been one of the main research subjects. Classical techniques, even with the help of computers, have, in a way, exhausted their potential and forced the appearance of new methods in order to attend the growing need of harder classifications. Examples of these new methods can be found in [3,12]. In [1] Arnol'd defined liftable vector fields and showed how they can be used for classification purposes. In fact, the new methods developed rely in many aspects in the computation of liftable vector fields. However, it is not easy at all to compute liftable vector fields in general. Liftable vector fields of a map germ are strongly related to the Derlog of the discriminant of the map germ (the module of tangent vector fields to the discriminant), which are of great importance in algebraic geometry besides singularity theory.

Here we consider $\mathscr{A}$-classification of map-germs $f:\left(\mathbb{K}^{n}, S\right) \rightarrow\left(\mathbb{K}^{p}, 0\right)$, where $\mathbb{K}=\mathbb{R}$ or $\mathbb{K}=\mathbb{C}, S \subset \mathbb{K}$ is a finite subset and the map-germ is either smooth when $\mathbb{K}=\mathbb{R}$ or

Work of both authors partially supported by MICINN Grant PGC2018-094889-B-I00 and GVA Grant AICO/2019/024.

R. Oset Sinha

Raul.Oset@uv.es

J. J. Nuño-Ballesteros

Juan.Nuno@uv.es

1 Departament de Matemàtiques, Universitat de València, Campus de Burjassot, 46100 Burjassot, Spain 
holomorphic when $\mathbb{K}=\mathbb{C}$. In [11], the authors gave a method to construct liftable vector fields for a certain class of map-germs $f$ which includes stable germs. They then showed how to obtain liftable vector fields of a finite singularity type germ $f$ from liftable vector fields of $F$, a stable unfolding of it. By finite singularity type, we mean a germ which admits a stable unfolding with a finite number of parameters. Furthermore, if $F$ is a one-parameter stable unfolding, they showed that any liftable vector field of $f$ comes from a liftable vector fields of $F$ by the previous construction. Although the class of map-germs which admit oneparameter stable unfoldings seems to be quite big amongst simple germs, it is still not fully understood when this is the case.

In this paper we generalize the result in [11], that is, we prove that all liftable vector fields of any finite singularity type map-germ can be obtained from the liftable vector fields of a stable unfolding of it, with no restriction on the number of parameters. The main result is that if $F$ is an $r$-parameter stable unfolding of $f$, then

$$
\operatorname{Lift}(f)=\pi_{1}\left(i^{*}(\operatorname{Lift}(F) \cap G)\right),
$$

where $\pi_{1}$ is the projection onto the first $p$ components, $i^{*}$ is the morphism induced by $i(X)=(X, 0)$ and $G$ is the submodule of $\theta_{p+r}$ generated by $\partial / \partial X_{k}$, with $1 \leq k \leq p$ and $\Lambda_{i} \partial / \partial \Lambda_{j}, 1 \leq i, j \leq r$ (here we denote by $(X, \Lambda)$ the coordinates in $\left.\mathbb{K}^{p} \times \mathbb{K}^{r}\right)$. As an application, we obtain the liftable vector fields for the family $H_{k}$ in Mond's list of simple germs $f:\left(\mathbb{K}^{2}, 0\right) \rightarrow\left(\mathbb{K}^{3}, 0\right)$ [9]. Another interesting corollary is that in the complex case and provided that either $n \leq p$ or $n>p>2$, then

$$
\operatorname{Derlog}(\Delta(f))=\pi_{1}\left(i^{*}(\operatorname{Derlog}(\Delta(F)) \cap G)\right),
$$

where $\Delta(f)$ and $\Delta(F)$ are the discriminants of $f$ and $F$ respectively. When $n>p=1$, this equality holds if and only if $f$ is quasihomogeneous (up to $\mathscr{A}$-equivalence) and when $n>p=2$ it is not known.

In the last section, we turn our attention to families of singularities which can be obtained by the method of augmentation. We show the relation between the liftable vector fields of a oneparameter stable unfolding of a map-germ and of its augmentations. We then use this result to prove the following: let $F$ be a one-parameter stable unfolding of $f:\left(\mathbb{C}^{n}, S\right) \rightarrow\left(\mathbb{C}^{p}, 0\right)$ with $p \leq n+1$ (except for $n>p \leq 2$ ) and let $A f$ be the augmentation of $f$ by $F$ and a quasihomogeneous function $\phi$, then

$$
\pi_{2}\left(i^{*}(\operatorname{Lift}(A f))\right)=\pi_{2}\left(i^{*}(\operatorname{Lift}(F))\right),
$$

where now $\pi_{2}$ is the projection onto the last component. What is meant here is that these two spaces are isomorphic, so the equality is understood as an equality of ideals of $\mathcal{O}_{p}$, the ring of smooth function germs in $p$-variables. This property allows us to use some results in $[2,12,13]$, where this condition is required.

\section{Notation}

We consider map-germs $f:\left(\mathbb{K}^{n}, S\right) \rightarrow\left(\mathbb{K}^{p}, 0\right)$, where $\mathbb{K}=\mathbb{R}$ or $\mathbb{K}=\mathbb{C}$, and $S \subset \mathbb{K}^{n}$ a finite subset. For simplicity, we will say that $f$ is smooth if it is smooth (i.e. $C^{\infty}$ ) when $\mathbb{K}=\mathbb{R}$ or holomorphic when $\mathbb{K}=\mathbb{C}$. We denote by $\mathcal{O}_{n}=\mathcal{O}_{\mathbb{K}^{n}, S}$ and $\mathcal{O}_{p}=\mathcal{O}_{\mathbb{K}^{p}, 0}$ the rings of smooth function germs in the source and target respectively and by $\theta_{n}=\theta_{\mathbb{K}^{n}, S}$ and $\theta_{p}=\theta_{\mathbb{K}^{p}, 0}$ the corresponding modules of vector field germs. The module of vector fields along $f$ will be denoted by $\theta(f)$. Associated with $\theta(f)$ we have two morphisms 
tf $: \theta_{n} \rightarrow \theta(f)$, given by $t f(\chi)=d f \circ \chi$, and $w f: \theta_{p} \rightarrow \theta(f)$, given by $w f(\eta)=\eta \circ f$. The $\mathscr{A}_{e}$-tangent space and the $\mathscr{A}_{e}$-codimension of $f$ are defined respectively as

$$
T \mathscr{A}_{e} f=t f\left(\theta_{n}\right)+w f\left(\theta_{p}\right), \quad \mathscr{A}_{e}-\operatorname{cod}(f)=\operatorname{dim}_{\mathbb{K}} \frac{\theta(f)}{T \mathscr{A}_{e} f} .
$$

It follows from Mather's infinitesimal stability criterion [8] that a germ is stable if and only if its $\mathscr{A}_{e}$-codimension is 0 . We refer to Wall's survey paper [14] and the book [10] for general background on the theory of singularities of mappings.

We will say that $f$ has finite singularity type if

$$
\operatorname{dim}_{\mathbb{K}} \frac{\theta(f)}{t f\left(\theta_{n}\right)+\left(f^{*} m_{p}\right) \theta_{n}}<\infty,
$$

where $m_{p}$ is the maximal ideal of $\mathcal{O}_{p}$ and $f^{*}: \mathcal{O}_{p} \rightarrow \mathcal{O}_{n}$ is the induced map. Another remarkable result of Mather is that $f$ has finite singularity type if and only if it admits an $r$-parameter stable unfolding (see [14]). We recall that an $r$-parameter unfolding of $f$ is another map-germ

$$
F:\left(\mathbb{K}^{n} \times \mathbb{K}^{r}, S \times\{0\}\right) \rightarrow\left(\mathbb{K}^{p} \times \mathbb{K}^{r}, 0\right)
$$

of the form $F(x, \lambda)=\left(f_{\lambda}(x), \lambda\right)$ and such that $f_{0}=f$.

Along the paper, we use the notation of small letters $x_{1}, \ldots, x_{n}, \lambda_{1}, \ldots, \lambda_{r}$ for the coordinates in $\mathbb{K}^{n} \times \mathbb{K}^{r}$ and capital letters $X_{1}, \ldots, X_{p}, \Lambda_{1}, \ldots, \Lambda_{r}$ for the coordinates in $\mathbb{K}^{p} \times \mathbb{K}^{r}$.

Definition 1 Let $f:\left(\mathbb{K}^{n}, S\right) \rightarrow\left(\mathbb{K}^{p}, 0\right)$ be a smooth map-germ.

(1) A vector field germ $\eta \in \theta_{p}$ is called liftable over $f$, if there exists $\xi \in \theta_{n}$ such that $d f \circ \xi=\eta \circ f$ (i.e., $t f(\xi)=w f(\eta)$ ). The set of vector field germs liftable over $f$ is denoted by $\operatorname{Lift}(f)$ and is an $\mathcal{O}_{p}$-submodule of $\theta_{p}$.

(2) We also define $\tilde{\tau}(f)=e v_{0}(\operatorname{Lift}(f))$, the subspace of $T_{0} \mathbb{K}^{p}$ given by the evaluation at the origin of elements of $\operatorname{Lift}(f)$.

When $f$ has finite $\mathscr{A}_{e}$-codimension, the space $\tilde{\tau}(f)$ can be interpreted geometrically as the tangent space to the isosingular locus (or analytic stratum in Mather's terminology) of $f$. This is the well defined manifold in the target along which the map $f$ is trivial. See $[3,6]$ for some basic properties of $\tilde{\tau}(f)$.

When $\mathbb{K}=\mathbb{C}$ and $f$ has finite singularity type, we always have the inclusion $\operatorname{Lift}(f) \subseteq$ $\operatorname{Der} \log (\Delta(f))$, where $\Delta(f)$ is the discriminant of $f$ (i.e., the image of non submersive points of $f$ ) and $\operatorname{Derlog}(\Delta(f))$ is the submodule of $\theta_{p}$ of vector fields which are tangent to $\Delta(f)$. Moreover, for all $(n, p)$ except for the case $n>p \leq 2$, we have the equality $\operatorname{Lift}(f)=\operatorname{Derlog}(\Delta(f))$ if $f$ has finite $\mathscr{A}_{e}$-codimension. This was proven in [1] for the $n=p$ case, in [4] for when $f$ is stable and in Proposition 8.8 and Remark 8.2 in [10] for all $(n, p)$ except for the case $n>p \leq 2$. If $n>p=1$ and $f$ has finite $\mathscr{A}_{e}$-codimension, $\operatorname{Lift}(f)=\operatorname{Derlog}(\Delta(f))$ if and only if $f$ is quasihomogeneous. If $n>p=2$ it is not known.

Definition 2 Let $h:\left(\mathbb{K}^{n}, S\right) \rightarrow\left(\mathbb{K}^{p}, 0\right)$ be a map-germ with a 1-parameter stable unfolding $H(x, \lambda)=\left(h_{\lambda}(x), \lambda\right)$. Let $g:\left(\mathbb{K}^{q}, 0\right) \rightarrow(\mathbb{K}, 0)$ be a function-germ. Then, the augmentation of $h$ by $H$ and $g$ is the map $A_{H, g}(h)$ given by $(x, z) \mapsto\left(h_{g(z)}(x), z\right)$. 


\section{Liftable vector fields and stable unfoldings}

In this section we show that all the liftable vector fields of any map-germ of finite singularity type can be obtained from the liftable vector fields of a stable unfolding (not necessarily versal) of it.

Theorem 1 Let $f:\left(\mathbb{K}^{n}, S\right) \longrightarrow\left(\mathbb{K}^{p}, 0\right)$ be a non-stable germ of finite singularity type and let $F$ be an $r$-parameter stable unfolding. Then,

$$
\operatorname{Lift}(f)=\pi_{1}\left(i^{*}(\operatorname{Lift}(F) \cap G)\right),
$$

where $\pi_{1}$ is the projection onto the first $p$ components, $i^{*}$ is the morphism induced by $i(X)=(X, 0)$ and $G$ is the submodule of $\theta_{p+r}$ generated by $\partial / \partial X_{k}$, with $1 \leq k \leq p$ and $\Lambda_{i} \partial / \partial \Lambda_{j}, 1 \leq i, j \leq r$.

Proof The proof is similar to the proof in Theorem 2 in [11]. We will prove it by a double inclusion. For simplicity, we set $\varphi=\pi_{1} \circ i^{*}: \theta_{p+r} \rightarrow \theta_{p}$.

Consider $\eta \in \operatorname{Lift}(F) \cap G$. There exists $\xi \in \theta_{n+r}$ such that $d F \circ \xi=\eta \circ F$. Evaluating this system of equations in $\lambda=0$ we have the following:

$$
\left(\frac{\partial f_{\lambda}}{\partial x}(x, 0)\right)\left(\begin{array}{c}
\xi_{1}(x, 0) \\
\vdots \\
\xi_{n}(x, 0)
\end{array}\right)+\left(\frac{\partial f_{\lambda}}{\partial \lambda}(x, 0)\right)\left(\begin{array}{c}
\xi_{n+1}(x, 0) \\
\vdots \\
\xi_{n+r}(x, 0)
\end{array}\right)=\left(\begin{array}{c}
\eta_{1}(F(x, 0)) \\
\vdots \\
\eta_{p}(F(x, 0))
\end{array}\right)
$$

and

$$
\xi_{n+k}(x, 0)=\eta_{p+k}(F(x, 0))=\eta_{p+k}(f(x), 0) \quad(1 \leq k \leq r) .
$$

Since $\eta \in G, \xi_{n+k}(x, 0)=\eta_{p+k}(f(x), 0)=0$, and so

$$
\left(\frac{\partial f_{\lambda}}{\partial x}(x, 0)\right)\left(\begin{array}{c}
\xi_{1}(x, 0) \\
\vdots \\
\xi_{n}(x, 0)
\end{array}\right)=\left(\begin{array}{c}
\eta_{1}(F(x, 0)) \\
\vdots \\
\eta_{p}(F(x, 0))
\end{array}\right),
$$

which means that $\varphi(\eta(X, \Lambda))=\left(\eta_{1}(X, 0), \ldots, \eta_{p}(X, 0)\right) \in \operatorname{Lift}(f)$.

Now let $\bar{\eta} \in \operatorname{Lift}(f)$. By definition, there exists a vector field $\bar{\xi} \in \theta_{n}$ such that $d f(\bar{\xi})=$ $\bar{\eta} \circ f$. Define $\eta(X, \Lambda)=(\bar{\eta}(X), 0)$ and $\xi(x, \lambda)=(\bar{\xi}(x), 0)$. Consider $\tilde{\eta}=\eta \circ F-d F(\xi) \in$ $\theta(F)$. We have $\widetilde{\eta}(x, 0)=(0,0)$ so by Hadamard's Lemma there exist vector fields $\widetilde{\eta}_{i} \in \theta(F)$ with $1 \leq i \leq r$ such that $\tilde{\eta}=\sum_{i=1}^{r} \Lambda_{i} \tilde{\eta}_{i}$.

Since $F$ is stable, there exist $\hat{\xi}_{i} \in \theta_{n+r}$ and $\hat{\eta}_{i} \in \theta_{p+r}$ for $1 \leq i \leq r$ such that $\tilde{\eta}_{i}=$ $d F\left(\hat{\xi}_{i}\right)+\hat{\eta}_{i} \circ F$ for all $i$. Therefore,

$$
\begin{aligned}
\eta \circ F-d F(\xi)=\tilde{\eta} & =\sum_{i=1}^{r} \Lambda_{i} \tilde{\eta}_{i}=\sum_{i=1}^{r} \Lambda_{i}\left(d F\left(\hat{\xi}_{i}\right)+\hat{\eta}_{i} \circ F\right) \\
& =d F\left(\sum_{i=1}^{r} \lambda_{i} \hat{\xi}_{i}\right)+\left(\sum_{i=1}^{r} \Lambda_{i} \hat{\eta}_{i}\right) \circ F
\end{aligned}
$$

Since $\eta$ is 0 in the last $r$ components, we have that $\eta-\sum_{i=1}^{r} \Lambda_{i} \hat{\eta}_{i} \in \operatorname{Lift}(F) \cap G$ and $\varphi\left(\eta-\sum_{i=1}^{r} \Lambda_{i} \hat{\eta}_{i}\right)=\bar{\eta}$. 
Remark 1 If $r=1$, then $G=\operatorname{Lift}(g)$, where $g:\left(\mathbb{K}^{p} \times \mathbb{K}, 0\right) \rightarrow\left(\mathbb{K}^{p} \times \mathbb{K}, 0\right)$ is the map germ $g(X, \Lambda)=\left(X, \Lambda^{2}\right)$ as was stated in Theorem 2 in [11]. However, if $r>1$ and $g:\left(\mathbb{K}^{p} \times \mathbb{K}^{r}, S\right) \rightarrow\left(\mathbb{K}^{p} \times \mathbb{K}^{r}, 0\right)$, where $S=\left\{z_{1}, \ldots, z_{r}\right\}$ and the branch $z_{i}$ is given by $g_{i}(X, \Lambda)=\left(X, \Lambda_{1}, \ldots, \Lambda_{i}^{2}, \ldots, \Lambda_{r}\right)$, then

$$
\operatorname{Lift}(g)=\mathcal{O}_{p+r}\left\{\frac{\partial}{\partial X_{k}}, 1 \leq k \leq p, \Lambda_{i} \frac{\partial}{\partial \Lambda_{i}}, 1 \leq i \leq r\right\} \varsubsetneqq G .
$$

When $\mathbb{K}=\mathbb{C}$ and $f$ has finite $\mathscr{A}_{e}$-codimension $\operatorname{Lift}(f)=\operatorname{Derlog}(\Delta(f))$ (unless $n>$ $p \leq 2$ ), so we have the following:

Corollary 1 Let $f:\left(\mathbb{C}^{n}, S\right) \longrightarrow\left(\mathbb{C}^{p}, 0\right)$ be a non-stable germ offinite $\mathscr{A}_{e}$-codimension such that either $n \leq p$ or $n>p>2$ and let $F$ be an $r$-parameter stable unfolding. Then,

$$
\operatorname{Derlog}(\Delta(f))=\pi_{1}\left(i^{*}(\operatorname{Derlog}(\Delta(F)) \cap G)\right) .
$$

Moreover, when $n>p=1$, the above equality holds if and only if $f$ is quasihomogeneous.

\subsection{Liftable vector fields of $\boldsymbol{H}_{k}$}

As an application of Theorem 1 we compute the liftable vector fields of a family of germs which do not admit a 1-parameter stable unfolding. Consider $H_{k}:\left(\mathbb{K}^{2}, 0\right) \rightarrow\left(\mathbb{K}^{3}, 0\right)$, from Mond's list [9] given by

$$
H_{k}(x, y)=\left(x, y^{3}, y^{3 k-1}+x y\right) \text {. }
$$

For any $k \geq 2, H_{k}$ admits a 2-parameter stable unfolding $\mathscr{A}$-equivalent to $F:\left(\mathbb{K}^{4}, 0\right) \rightarrow$ $\left(\mathbb{K}^{5}, 0\right)$,

$$
F\left(u_{1}, v_{1}, v_{2}, y\right)=\left(u_{1}, v_{1}, v_{2}, y^{3}+u_{1} y, v_{1} y+v_{2} y^{2}\right),
$$

which is versal for $k=2$. In fact, the change of variables in the source $v_{2}^{\prime}=v_{2}-y^{3 k-3}$, the change $G_{k}\left(U_{1}, V_{1}, V_{2}, W_{1}, W_{2}\right)=\left(U_{1}, V_{1}, V_{2}-W_{1}^{k-1}, W_{1}, W_{2}\right)$ in the target, and a further change in the source, show that $F$ is $\mathscr{A}$-equivalent to

$$
F_{k}\left(u_{1}, v_{1}, v_{2}, y\right)=\left(u_{1}, v_{1}, v_{2}, y^{3}+u_{1} y, v_{1} y+v_{2} y^{2}+\psi\left(u_{1}, y\right) y^{2}+y^{3 k-1}\right),
$$

for some function $\psi$ such that $\psi(0, y)=0$. So $F_{k}$ is a 2-parameter stable unfolding of $H_{k}$.

In this case,

$$
G=\mathcal{O}_{3+2}\left\{U_{1} \frac{\partial}{\partial U_{1}}, V_{2} \frac{\partial}{\partial U_{1}}, U_{1} \frac{\partial}{\partial V_{2}}, V_{2} \frac{\partial}{\partial V_{2}}, \frac{\partial}{\partial V_{1}}, \frac{\partial}{\partial W_{1}}, \frac{\partial}{\partial W_{2}}\right\},
$$

and by Theorem 1, $\operatorname{Lift}\left(H_{k}\right)=\varphi\left(\operatorname{Lift}\left(F_{k}\right) \cap G\right)$, where $\varphi=\pi_{1} \circ i^{*}$.

In Example 3.4 in [7], the generators for $\operatorname{Lift}(F)$ are calculated. We show these vector fields as matrices for simplicity:

$$
\eta_{e}=\left(\begin{array}{c}
2 U_{1} \\
2 V_{1} \\
V_{2} \\
3 W_{1} \\
3 W_{2}
\end{array}\right), \eta_{1}^{1,2,3}=\left(\begin{array}{c}
4 U_{1}^{2} \\
-3 U_{1} V_{1}+3 V_{2} W_{1} \\
-5 U_{1} V_{2}-3 W_{2} \\
6 U_{1} W_{1} \\
-3 V_{1} W_{1}+2 U_{1} W_{2}
\end{array}\right),\left(\begin{array}{c}
6 U_{1} \\
-3 V_{1} \\
-6 V_{2} \\
9 W_{1} \\
0
\end{array}\right),\left(\begin{array}{c}
9 V_{1} \\
-6 V_{2}^{2} \\
0 \\
9 W_{2}+3 U_{1} V_{2} \\
3 V_{1} V_{2}
\end{array}\right),
$$




$$
\eta_{2}^{1,2,3}=\left(\begin{array}{c}
0 \\
-3 U_{1} V_{2}-3 W_{2} \\
3 V_{1} \\
0 \\
-3 V_{2} W_{1}
\end{array}\right),\left(\begin{array}{c}
-9 W_{1} \\
2 U_{1} V_{2} \\
-3 V_{1} \\
2 U_{1}^{2} \\
6 V_{2} W_{1}+2 U_{1} V_{1}
\end{array}\right),\left(\begin{array}{c}
-9 W_{2}-3 U_{1} V_{2} \\
-3 V_{1} V_{2} \\
0 \\
3 U_{1} V_{1} \\
6 V_{2} W_{2}+3 V_{1}^{2}
\end{array}\right)
$$

In [11, Lemma 6.1], it is shown that if two maps $f$ and $g$ are $\mathscr{A}$-equivalent, i.e. there exist $h$ and $H$ diffeomorphisms in source and target such that $f=H \circ g \circ h$, then the map $L_{f, g}: \operatorname{Lift}(g) \rightarrow \operatorname{Lift}(f)$ such that $L_{f, g}(\eta)=d H \circ \eta \circ H^{-1}$ is a bijection. We therefore can obtain $\operatorname{Lift}\left(F_{k}\right)$ from $\operatorname{Lift}(F)$ by composing its generators by $G_{k}^{-1}$ on the right and multiplying by $d G_{k}$ on the left.

$$
\begin{aligned}
& \eta_{k e}=\left(\begin{array}{c}
2 U_{1} \\
2 V_{1} \\
V_{2}-(3 k-4) W_{1}^{k-1} \\
3 W_{1} \\
3 W_{2}
\end{array}\right), \eta_{k 1}^{1}=\left(\begin{array}{c}
4 U_{1}^{2} \\
-3 U_{1} V_{1}+3 V_{2} W_{1}+3 W_{1}^{k} \\
-5 U_{1} V_{2}-(6 k-1) U_{1} W_{1}^{k-1}-3 W_{2} \\
6 U_{1} W_{1} \\
-3 V_{1} W_{1}+2 U_{1} W_{2}
\end{array}\right), \\
& \eta_{k 1}^{2}=\left(\begin{array}{c}
6 U_{1} \\
-3 V_{1} \\
-6 V_{2}-(9 k-3) W_{1}^{k-1} \\
9 W_{1} \\
0
\end{array}\right), \eta_{k 2}^{1}=\left(\begin{array}{c}
0 \\
-3 U_{1} V_{2}-3 U_{1} W_{1}^{k-1}-3 W_{2} \\
3 V_{1} \\
0 \\
-3 V_{2} W_{1}-3 W_{1}^{k}
\end{array}\right), \\
& \eta_{k 1}^{3}=\left(\begin{array}{c}
9 V_{1} \\
-6 V_{2}^{2}-12 V_{2} W_{1}^{k-1}-6 W_{1}^{2 k-2} \\
-9(k-1) W_{1}^{k-2} W_{2}-3(k-1) U_{1} V_{2} W_{1}^{k-2}-3(k-1) U_{1} W_{1}^{2 k-3} \\
9 W_{2}+3 U_{1} V_{2}+3 U_{1} W_{1}^{k-1} \\
3 V_{1} V_{2}+3 V_{1} W_{1}^{k-1}
\end{array}\right), \\
& \eta_{k 2}^{2}=\left(\begin{array}{c}
-9 W_{1} \\
2 U_{1} V_{2}+2 U_{1} W_{1}^{k-1} \\
-3 V_{1}-2(k-1) U_{1}^{2} W_{1}^{k-2} \\
2 U_{1}^{2} \\
6 V_{2} W_{1}+6 W_{1}^{k}+2 U_{1} V_{1}
\end{array}\right), \eta_{k 2}^{3}=\left(\begin{array}{c}
-9 W_{2}-3 U_{1} V_{2}-3 U_{1} W_{1}^{k-1} \\
-3 V_{1} V_{2}-3 V_{1} W_{1}^{k-1} \\
-3(k-1) U_{1} V_{1} W_{1}^{k-2} \\
3 U_{1} V_{1} \\
6 V_{2} W_{2}+6 W_{1}^{k-1} W_{2}+3 V_{1}^{2}
\end{array}\right) .
\end{aligned}
$$

The module $\operatorname{Lift}\left(F_{k}\right) \cap G$ is formed by vector fields of type

$$
\eta=\tilde{\alpha}_{1} \eta_{k e}+\tilde{\alpha}_{2} \eta_{k 1}^{1}+\tilde{\alpha}_{3} \eta_{k 1}^{2}+\tilde{\alpha}_{4} \eta_{k 2}^{1}+\tilde{\alpha}_{5} \eta_{k 1}^{3}+\tilde{\alpha}_{6} \eta_{k 2}^{2}+\tilde{\alpha}_{7} \eta_{k 2}^{3},
$$

such that the first and third components are divisible by $U_{1}$ or $V_{2}$, where the $\tilde{\alpha}_{i}$ are functions in $U_{1}, V_{1}, V_{2}, W_{1}$ and $W_{2}$. This means that the first and third components evaluated at $U_{1}=V_{2}=0$ are equal to 0 , which yields two conditions on $\alpha_{i}\left(V_{1}, W_{1}, W_{2}\right)=$ $\tilde{\alpha}_{i}\left(0, V_{1}, 0, W_{1}, W_{2}\right)$ :

$$
\begin{aligned}
& 9 \alpha_{5} V_{1}-9 \alpha_{6} W_{1}-9 \alpha_{7} W_{2}=0,-(3 k-4) \alpha_{1} W_{1}^{k-1}-3 \alpha_{2} W_{2} \\
& -(9 k-3) \alpha_{3} W_{1}^{k-1}+3 \alpha_{4} V_{1}-9(k-1) \alpha_{5} W_{1}^{k-2} W_{2}-3 \alpha_{6} V_{1}=0 .
\end{aligned}
$$

Now, set $\beta=-(3 k-4) \alpha_{1}-(9 k-3) \alpha_{3}$, so that $\alpha_{3}=-\frac{1}{9 k-3} \beta-\frac{3 k-4}{9 k-3} \alpha_{1}$. The conditions can be rewritten as

$$
\alpha_{5} V_{1}=\alpha_{6} W_{1}+\alpha_{7} W_{2},
$$




$$
\alpha_{4} V_{1}=-\frac{1}{3} \beta W_{1}^{k-1}+\alpha_{2} W_{2}+3(k-1) \alpha_{5} W_{1}^{k-2} W_{2}+\alpha_{6} V_{1} .
$$

By Theorem 1, the liftable vector fields of $H_{k}$ are obtained from the second, fourth and fifth components vector fields of the type of $\eta$ evaluated at $U_{1}=V_{2}=0$. That is,

$$
\begin{aligned}
\operatorname{Lift}\left(H_{k}\right)= & \left\{\alpha_{1}\left(2 V_{1} \frac{\partial}{\partial V_{1}}+3 W_{1} \frac{\partial}{\partial W_{1}}+3 W_{2} \frac{\partial}{\partial W_{2}}\right)+\alpha_{2}\left(3 W_{1}^{k} \frac{\partial}{\partial V_{1}}-3 V_{1} W_{1} \frac{\partial}{\partial W_{2}}\right)\right. \\
& +\alpha_{3}\left(-3 V_{1} \frac{\partial}{\partial V_{1}}+9 W_{1} \frac{\partial}{\partial W_{1}}\right)+\alpha_{4}\left(-3 W_{2} \frac{\partial}{\partial V_{1}}-3 W_{1}^{k} \frac{\partial}{\partial W_{2}}\right) \\
& +\alpha_{5}\left(-6 W_{1}^{2 k-2} \frac{\partial}{\partial V_{1}}+9 W_{2} \frac{\partial}{\partial W_{1}}+3 V_{1} W_{1}^{k-1} \frac{\partial}{\partial W_{2}}\right)+\alpha_{6}\left(6 W_{1}^{k} \frac{\partial}{\partial W_{2}}\right) \\
& \left.+\alpha_{7}\left(-3 V_{1} W_{1}^{k-1} \frac{\partial}{\partial V_{1}}+\left(6 W_{1}^{k-1} W_{2}+3 V_{1}^{2}\right) \frac{\partial}{\partial W_{2}}\right)\right\},
\end{aligned}
$$

where the $\alpha_{i}$ are germs of functions.

We use conditions (1) and (2) to eliminate the functions $\alpha_{4}, \alpha_{5}$. Then, after some easy simplifications in the obtained generators, we arrive to the following:

Theorem 2 The module of liftable vector fields over the map germs $H_{k}:\left(\mathbb{K}^{2}, 0\right) \rightarrow\left(\mathbb{K}^{3}, 0\right)$, given by $H_{k}(x, y)=\left(x, y^{3}, y^{3 k-1}+x y\right)$ is generated by the following vector fields

$$
\begin{gathered}
\left(\begin{array}{c}
(3 k-2) V_{1} \\
3 W_{1} \\
(3 k-1) W_{2}
\end{array}\right),\left(\begin{array}{c}
V_{1}^{2}+(3 k-1) W_{1}^{k-1} W_{2} \\
-3 V_{1} W_{1} \\
(3 k-1) W_{1}^{2 k-1}
\end{array}\right),\left(\begin{array}{c}
W_{2}^{2}-V_{1} W_{1}^{k} \\
0 \\
V_{1}^{2} W_{1}+W_{1}^{k} W_{2}
\end{array}\right), \\
\left(\begin{array}{c}
(3 k-1) W_{1}^{2 k-1}+V_{1} W_{2} \\
-3 W_{1} W_{2} \\
-(3 k-1) V_{1} W_{1}^{k}
\end{array}\right),\left(\begin{array}{c}
-(3 k-1) W_{1}^{2 k-2} W_{2}-V_{1}^{2} W_{1}^{k-1} \\
3 W_{2}^{2} \\
3 k V_{1} W_{1}^{k-1} W_{2}+V_{1}^{3}
\end{array}\right) .
\end{gathered}
$$

Remark 2 In fact, these vector fields can be obtained by applying $\varphi$ to the following vector fields in $\operatorname{Lift}\left(F_{k}\right) \cap G$ :

$$
\begin{aligned}
& (9 k-3) \eta_{k e}-(3 k-4) \eta_{k 1}^{2}, \\
& 3 V_{1} \eta_{k 1}^{2}+(9 k-3) W_{1}^{k-1} \eta_{k 2}^{1}, \\
& 2 V_{1} \eta_{k 1}^{1}+W_{2}\left(\eta_{k 2}^{1}-\eta_{k 2}^{2}\right)+W_{1} \eta_{k 2}^{3}, \\
& W_{1} \eta_{k 1}^{3}-3(k-1) W_{1}^{k-1} \eta_{k 1}^{1}+V_{1}\left(\eta_{k 2}^{1}+\eta_{k 2}^{2}\right), \\
& W_{2} \eta_{k 1}^{3}+V_{1} \eta_{k 2}^{3}-3(k-1) W_{1}^{k-2} W_{2} \eta_{k 2}^{1} .
\end{aligned}
$$

\section{Liftable vector fields and augmentations}

In this section, we restrict ourselves to the complex case $\mathbb{K}=\mathbb{C}$. Suppose $f:\left(\mathbb{C}^{n}, S\right) \longrightarrow$ $\left(\mathbb{C}^{p}, 0\right)$ admits a one-parameter stable unfolding $F(x, \lambda)=\left(f_{\lambda}(x), \lambda\right)$. Let $\phi:(\mathbb{C}, 0) \rightarrow$ $(\mathbb{C}, 0), \phi \neq 0$, and let $A f(x, z)=\left(f_{\phi(z)}(x), z\right)=(X, Z)$ be the augmentation of $f$ by $F$ and $\phi$. We shall establish a relation between the liftable vector fields of an augmentation $A f$ and those of the stable unfolding $F$.

As we pointed out in Sect. 2 , if $g$ is a complex analytic germ of finite $\mathscr{A}_{e}$-codimension and $n \leq p$ or $n>p>2$, then $\operatorname{Lift}(g)=\operatorname{Derlog}(\Delta(g))$. When $p \leq n+1, \Delta(g)$ is always a hypersurface in $\left(\mathbb{C}^{p}, 0\right)$, hence $\operatorname{Derlog}(h) \subset \operatorname{Derlog}(\Delta(g))$, where $h$ is the reduced defining equation for $\Delta(g)$ and

$$
\operatorname{Derlog}(h)=\left\{\eta \in \theta_{p}: \eta(h)=0\right\} .
$$

Furthermore, if $g$ is quasihomogeneous $\operatorname{Derlog}(\Delta(g))=\langle e\rangle \oplus \operatorname{Derlog}(h)$, where $e$ is the Euler vector field (see for instance [5]). 
Lemma 1 Let $H(X, \Lambda)$ be a defining equation of $\Delta(F)$, then we can take $h(X, Z)=$ $H(X, \phi(Z))$ as a defining equation of $\Delta(A f)$.

Proof Let $F(x, \lambda)=\left(f_{\lambda}(x), \lambda\right)$. The critical set of $F$ is given by $C_{F}=\{(x, \lambda) \in$ $\mathbb{C}^{n} \times \mathbb{C}: F$ is not a submersion in $\left.(x, \lambda)\right\}$ and this is equal to $\left\{(x, \lambda) \in \mathbb{C}^{n} \times \mathbb{C}\right.$ : $f_{\lambda}$ is not a submersion in $x$ \}. Notice that when $n<p$ the critical set is the whole space and instead of discriminant we are considering the image. Now, $\Delta(F)=F\left(C_{F}\right)$ is given by $H(X, \Lambda)=0$, so for all $(x, \lambda)$ such that $f_{\lambda}$ is not a submersion in $x$ we have $H\left(f_{\lambda}(x), \lambda\right)=0$. Changing $\lambda$ for $\phi(z)$ we get that for all $(x, z)$ such that $f_{\phi(z)}$ is not a submersion in $x$ we have $H\left(f_{\phi(z)}(x), \phi(z)\right)=0$.

Similarly $C_{A f}=\left\{(x, z) \in \mathbb{C}^{n} \times \mathbb{C}: f_{\phi(z)}\right.$ is not a submersion in $\left.x\right\}$. Setting $h(X, Z)=$ $H(X, \phi(Z))$ we get that

$$
h(A f(x, z))=h\left(f_{\phi(z)}(x), z\right)=H\left(f_{\phi(z)}(x), \phi(z)\right)=0,
$$

so $h(X, Z)$ is a defining equation of $\Delta(A f)$.

In particular,

$$
\frac{\partial h}{\partial X_{i}}(X, Z)=\frac{\partial H}{\partial X_{i}}(X, \phi(Z)), i=1, \ldots, p, \quad \frac{\partial h}{\partial Z}(X, Z)=\frac{\partial H}{\partial \Lambda}(X, \phi(Z)) \phi^{\prime}(Z) .
$$

Given a vector field $\eta(X, \Lambda)=\sum_{i=1}^{p} \eta_{i}(X, \Lambda) \frac{\partial}{\partial X_{i}}+\eta_{p+1}(X, \Lambda) \frac{\partial}{\partial \Lambda} \in \theta_{p+1}$, we set:

$$
\tilde{\eta}(X, Z)=\sum_{i=1}^{p} \eta_{i}(X, \phi(Z)) \phi^{\prime}(Z) \frac{\partial}{\partial X_{i}}+\eta_{p+1}(X, \phi(Z)) \frac{\partial}{\partial Z} \in \theta_{p+1} .
$$

We have the following two lemmas:

Lemma 2 If $\eta \in \operatorname{Derlog}(\Delta(F))$, then $\tilde{\eta} \in \operatorname{Derlog}(\Delta(A f))$. Furthermore, suppose that $\eta_{p+1}(X, 0)=0$, then $\frac{\widetilde{\eta}}{\phi^{\prime}} \in \operatorname{Derlog}(\Delta(A f))$.

Proof If $\eta \in \operatorname{Derlog}(\Delta(F)), \eta(H)=\alpha H$ for some $\alpha \in \mathcal{O}_{p+1}$. That is,

$$
\sum_{i=1}^{p} \eta_{i}(X, \Lambda) \frac{\partial H}{\partial X_{i}}(X, \Lambda)+\eta_{p+1}(X, \Lambda) \frac{\partial H}{\partial \Lambda}(X, \Lambda)=\alpha(X, \Lambda) H(X, \Lambda) .
$$

Substituting $\Lambda=\phi(Z)$ and multiplying by $\phi^{\prime}(Z)$ yields

$$
\begin{aligned}
& \sum_{i=1}^{p} \eta_{i}(X, \phi(Z)) \phi^{\prime}(Z) \frac{\partial H}{\partial X_{i}}(X, \phi(Z))+\eta_{p+1}(X, \phi(Z)) \phi^{\prime}(Z) \frac{\partial H}{\partial \Lambda}(X, \phi(Z)) \\
& \quad=\alpha(X, \phi(Z)) \phi^{\prime}(Z) H(X, \phi(Z)) .
\end{aligned}
$$

Hence, $\tilde{\eta}(h)=\phi^{\prime} \widetilde{\alpha} h$, where $\widetilde{\alpha}(X, Z)=\alpha(X, \phi(Z))$ and thus $\tilde{\eta} \in \operatorname{Derlog}(\Delta(A f))$.

On the other hand, if $\eta_{p+1}(X, 0)=0$ we can write $\eta_{p+1}=\Lambda a$, for some $a \in \mathcal{O}_{p+1}$. We also put $\phi=b \phi^{\prime}$, for some $b \in \mathcal{O}_{1}$. We have:

$$
\eta_{p+1}(X, \phi(Z))=\phi(Z) a(X, \phi(Z))=b(Z) \phi^{\prime}(Z) a(X, \phi(Z)) .
$$

Thus $\tilde{\eta}$ is divisible by $\phi^{\prime}$ and $\frac{\widetilde{\eta}(h)}{\phi^{\prime}}=\widetilde{\alpha} h$, so $\frac{\widetilde{\eta}}{\phi^{\prime}} \in \operatorname{Derlog}(\Delta(A f))$. 
Lemma 3 Suppose $\phi \neq 0$ is quasihomogeneous and let $\bar{\eta} \in \operatorname{Derlog}(\Delta(A f))$. Then there exists $\eta \in \operatorname{Derlog}(\Delta(F))$ such that $\tilde{\eta}=\bar{\eta}+\varphi$ with $\varphi \in \operatorname{Derlog}(\Delta(A f))$. Furthermore, if $\bar{\eta}_{p+1}(X, 0)=0$ then there exists $\eta \in \operatorname{Derlog}(\Delta(F))$ such that $\frac{\widetilde{\eta}}{\phi^{\prime}}=\bar{\eta}+\varphi$, with $\varphi \in$ $\operatorname{Derlog}(\Delta(A f))$.

Proof We put $\bar{\eta}_{p+1}(X, Z)=Z \psi(X, Z)+\beta(X)$, for some functions $\psi, \beta$, hence

$$
\bar{\eta}(X, Z)=\sum_{i=1}^{p} \bar{\eta}_{i}(X, Z) \frac{\partial}{\partial X_{i}}+(Z \psi(X, Z)+\beta(X)) \frac{\partial}{\partial Z} .
$$

For simplicity we suppose $\bar{\eta} \in \operatorname{Derlog}(h)$ and comment on the general case later. So, we have

$$
\sum_{i=1}^{p} \bar{\eta}_{i}(X, Z) \frac{\partial h}{\partial X_{i}}(X, Z)=-(Z \psi(X, Z)+\beta(X)) \frac{\partial h}{\partial Z}(X, Z),
$$

which means

$$
\begin{aligned}
& \sum_{i=1}^{p} \bar{\eta}_{i}(X, Z) \frac{\partial H}{\partial X_{i}}(X, \phi(Z))+Z \psi(X, Z) \phi^{\prime}(Z) \frac{\partial H}{\partial \Lambda}(X, \phi(Z)) \\
& =-\beta(X) \frac{\partial H}{\partial \Lambda}(X, \phi(Z)) \phi^{\prime}(Z) .
\end{aligned}
$$

Since $\phi(Z)=Z^{k}$ and $\phi^{\prime}(Z)=k Z^{k-1}$, the right hand side of this equality can be seen as a series in $Z$ of type

$$
c_{k-1}(X) Z^{k-1}+c_{2 k-1}(X) Z^{2 k-1}+c_{3 k-1}(X) Z^{3 k-1}+\cdots
$$

On the other hand, for $i=1, \ldots, p$,

$$
\begin{aligned}
\bar{\eta}_{i}(X, Z) & =a_{i 0}(X)+a_{i 1}(X) Z+a_{i 2}(X) Z^{2}+\cdots, \\
\frac{\partial H}{\partial X_{i}}(X, \phi(Z)) & =b_{i 0}(X)+b_{i k}(X) Z^{k}+b_{i, 2 k}(X) Z^{2 k}+\cdots
\end{aligned}
$$

Analogously for $Z \psi(X, Z)$ and $\frac{\partial H}{\partial \Lambda}(X, \phi(Z))$. By identifying the coefficients at both sides of the equality, the left hand side has a part equal to 0 (the sum of the coefficients whose terms do not appear in the right hand side) and the rest is used to rewrite the equality

$$
\begin{aligned}
& \sum_{i=1}^{p} A_{i}(X, \phi(Z)) \phi^{\prime}(Z) \frac{\partial H}{\partial X_{i}}(X, \phi(Z))+A_{p+1}(X, \phi(Z)) \phi^{\prime}(Z) \frac{\partial H}{\partial \Lambda}(X, \phi(Z)) \\
& \quad=-\beta(X) \phi^{\prime}(Z) \frac{\partial H}{\partial \Lambda}(X, \phi(Z)),
\end{aligned}
$$

for some functions $A_{i}, i=1, \ldots, p+1$. The part which was equal to 0 corresponds to a certain $\varphi \in \operatorname{Derlog}(h)$.

Dividing the previous equation by $\phi^{\prime}(Z)$ and substituting $\Lambda=\phi(Z)$ (since $\phi(Z)=Z^{k}$, $\phi$ is surjective), we obtain

$$
\sum_{i=1}^{p} A_{i}(X, \Lambda) \frac{\partial H}{\partial X_{i}}(X, \Lambda)+\left(A_{p+1}(X, \Lambda)+\beta(X)\right) \frac{\partial H}{\partial \Lambda}(X, \Lambda)=0
$$


which means that

$$
\eta=\sum_{i=1}^{p} A_{i} \frac{\partial}{\partial X_{i}}+\left(A_{p+1}+\beta\right) \frac{\partial}{\partial Z} \in \operatorname{Derlog}(H)
$$

and $\tilde{\eta}=\bar{\eta}-\varphi$.

If $\bar{\eta} \notin \operatorname{Derlog}(h)$, that is $\bar{\eta}(h)=\alpha(X, Z) h(X, Z)=\alpha(X, Z) H(X, \phi(Z))$, in the same way as above we would take only the part of $\alpha(X, Z) H(X, \phi(Z))$ in the powers $Z^{n k-1}$, that is $\gamma(X, \phi(Z)) \phi^{\prime}(Z) H(X, \phi(Z))$ and proceed in the same way.

Second, suppose that $\bar{\eta}_{p+1}(X, 0)=0$, so $\beta=0$. Then $\bar{\eta}(h)=\alpha h$ yields

$$
\sum_{i=1}^{p} \bar{\eta}_{i}(X, Z) \frac{\partial H}{\partial X_{i}}(X, \phi(Z))+Z \psi(X, Z) \phi^{\prime}(Z) \frac{\partial H}{\partial \Lambda}(X, \phi(Z))=\alpha(X, Z) H(X, \phi(Z)) .
$$

If we take only the terms in $Z^{n k}$ with $n \in\{0,1, \ldots\}$ on both sides of the equation we are left with

$$
\begin{aligned}
& \sum_{i=1}^{p} A_{i}(X, \phi(Z)) \frac{\partial H}{\partial X_{i}}(X, \phi(Z))+Z A_{p+1}(X, \phi(Z)) \phi^{\prime}(Z) \frac{\partial H}{\partial \Lambda}(X, \phi(Z)) \\
& \quad=\gamma(X, \phi(Z)) H(X, \phi(Z)) .
\end{aligned}
$$

Notice that $Z \phi^{\prime}(Z)=k Z^{k}=k \phi(Z)$, so if we substitute $\Lambda=\phi(Z)$, we get that

$$
\eta=\sum_{i=1}^{p} A_{i} \frac{\partial}{\partial X_{i}}+k \Lambda A_{p+1} \frac{\partial}{\partial \Lambda} \in \operatorname{Derlog}(\Delta(F)) .
$$

Remark 3 Lemmas 2 and 3 imply that the number of generators of $\operatorname{Derlog}(\Delta(A f)$ ) (and therefore of $\operatorname{Lift}(A f))$ is greater than or equal to the number of generators of $\operatorname{Derlog}(\Delta(F))$ $(\operatorname{Lift}(F)$ resp.). Notice too that if $\eta \in \operatorname{Derlog}(H)$, then $\tilde{\eta} \in \operatorname{Derlog}(h)$.

Example 1 Consider $f:\left(\mathbb{C}^{2}, 0\right) \rightarrow\left(\mathbb{C}^{2}, 0\right)$ given by $f(x, y)=\left(x^{4}+y x, y\right)$, its 1-parameter stable unfolding $F(x, y, z)=\left(x^{4}+y x+z x^{2}, y, z\right)$ and the augmentations by $\phi(z)=z^{k}$, $A^{k} f(x, y, z)=\left(x^{4}+y x+z^{k} x^{2}, y, z\right)$. The defining equation of the discriminant is

$$
h(X, Y, Z)=256 X^{3}+27 Y^{4}+144 X Y^{2} Z^{k}+128 X^{2} Z^{2 k}+4 Y^{2} Z^{3 k}+16 X Z^{4 k} .
$$

When $k=1$ we have $H$. It can be seen (see [1] for example) that $\operatorname{Lift}(F)=\left\langle\eta^{1}, \eta^{2}, \eta^{3}\right\rangle$, where

$$
\begin{aligned}
& \eta^{1}=4 X \frac{\partial}{\partial X}+3 Y \frac{\partial}{\partial Y}+2 Z \frac{\partial}{\partial Z}, \\
& \eta^{2}=-\left(9 Y^{2}+16 X Z\right) \frac{\partial}{\partial X}+12 Y Z \frac{\partial}{\partial Y}+\left(48 X+4 Z^{2}\right) \frac{\partial}{\partial Z}, \\
& \left.\eta^{3}=Y Z \frac{\partial}{\partial X}-\left(8 X+2 Z^{2}\right) \frac{\partial}{\partial Y}+6 Y \frac{\partial}{\partial Z}\right\rangle .
\end{aligned}
$$

On the other hand, $\operatorname{Lift}\left(A^{k} f\right)=\left\langle\widetilde{\eta}^{1}, \widetilde{\eta}^{2}, \widetilde{\eta}^{3}, \widetilde{\eta}^{4}\right\rangle$, where

$$
\tilde{\eta}^{1}=4 k X \frac{\partial}{\partial X}+3 k Y \frac{\partial}{\partial Y}+2 Z \frac{\partial}{\partial Z},
$$




$$
\begin{aligned}
\tilde{\eta}^{2}= & -\left(9 k Y^{2} Z^{k-1}+16 k X Z^{2 k-1}\right) \frac{\partial}{\partial X}+12 k Y Z^{2 k-1} \frac{\partial}{\partial Y}+\left(48 X+4 Z^{2 k}\right) \frac{\partial}{\partial Z}, \\
\tilde{\eta}^{3}= & k Y Z^{2 k-1} \frac{\partial}{\partial X}-\left(8 k X Z^{k-1}+2 k Z^{3 k-1}\right) \frac{\partial}{\partial Y}+6 Y \frac{\partial}{\partial Z} \\
\tilde{\eta}^{4}= & -\left(9 k Y^{3}+24 k X Y Z^{k}\right) \frac{\partial}{\partial X}+\left(64 k X^{2}+12 k Y^{2} Z^{k}+16 k X Z^{2 k}\right) \frac{\partial}{\partial Y} \\
& +4 Y Z^{k+1} \frac{\partial}{\partial Z} .
\end{aligned}
$$

Following Lemma 2, if we substitute $Z$ by $Z^{k}$ in $\eta^{2}$ and $\eta^{3}$ and multiply their first two components by $k Z^{k-1}$ we obtain $\widetilde{\eta}^{2}$ and $\widetilde{\eta}^{3}$. Now consider $\xi=k Y \eta^{2}(X, Y, Z)-$ $8 k X \eta^{3}(X, Y, Z)$. Since $\xi_{3}(X, Y, 0)=0$, again by Lemma 2 , substituting $Z$ by $Z^{k}$ and then dividing the last component by $\phi^{\prime}(Z)=k Z^{k-1}$ gives $\widetilde{\eta}^{4}$. Finally, if we take $k \eta^{1}(X, Y, Z)$, substitute $Z$ by $Z^{k}$ and then divide the last component by $\phi^{\prime}(Z)=k Z^{k-1}$ we get $\widetilde{\eta}^{1}$.

In [12] the operation of simultaneous augmentation and concatenation was defined as the multigerm defined by $\{A f, g\}$ where $g$ is a fold map or an immersion. The authors proved a formula relating the $\mathscr{A}_{e}$-codimensions of $\{A f, g\}$ and of $f$ under a certain condition, namely that $\pi_{2}\left(i^{*}(\operatorname{Lift}(A f))\right)=\pi_{2}\left(i^{*}(\operatorname{Lift}(F))\right)$ where $\pi_{2}$ is the projection onto the last component and $i$ is the immersion $i(X)=(X, 0)$. Here the equality stands for equality of ideals in $\mathcal{O}_{p}$. This condition appears again in [2] and in [13] where the authors say that all examples they have studied satisfy this condition but they were not able to prove it. We will use Theorem 1 and Lemmas 2, 3 in order to give a proof of this fact when the augmenting function $\phi$ is quasihomogeneous.

Theorem 3 Let $f:\left(\mathbb{C}^{n}, S\right) \longrightarrow\left(\mathbb{C}^{p}, 0\right)$ be a map germ of finite codimension with $p \leq n+1$ (except for $n>p \leq 2)$ which admits a one-parameter stable unfolding $F$. Let $\phi:(\mathbb{C}, 0) \rightarrow$ $(\mathbb{C}, 0)$ be a quasihomogeneous function and let $A f$ be the augmentation of $f$ by $F$ and $\phi$. Then,

$$
\pi_{2}\left(i^{*}(\operatorname{Lift}(A f))\right)=\pi_{2}\left(i^{*}(\operatorname{Lift}(F))\right),
$$

as ideals of $\mathcal{O}_{p}$.

Proof Let us see that $\pi_{2}\left(i^{*}(\operatorname{Lift}(F)) \subseteq \pi_{2}\left(i^{*}(\operatorname{Lift}(A f))\right)\right.$. Suppose $F(x, \lambda)=\left(f_{\lambda}(x), \lambda\right)$ and consider

$$
A F(x, z, \mu)=\left(f_{\phi(z)+\mu}(x), z, \mu\right)=(X, Z, M),
$$

the one-parameter stable unfolding of $A f$ and the immersion $i_{1}(X, Z)=(X, Z, 0)$. Then, by Theorem 1,

$$
\operatorname{Lift}(A f)=\varphi(\operatorname{Lift}(A F) \cap G)=\pi_{1}\left(i_{1}^{*}(\operatorname{Lift}(A F) \cap G)\right),
$$

where $G=\mathcal{O}_{p+1+1}\left\{\frac{\partial}{\partial X_{1}}, \ldots, \frac{\partial}{\partial X_{p}}, \frac{\partial}{\partial Z}, M \frac{\partial}{\partial M}\right\}$ and $\pi_{1}$ is the projection onto the first $p+1$ components. On the other hand, $A F$ is $\mathcal{A}$-equivalent to a trivial one-parameter unfolding of $F$, and if we consider the immersion $i_{1}^{\prime}(X, M)=(X, 0, M)$, then, by Theorem 1 ,

$$
\operatorname{Lift}(F)=\varphi^{\prime}\left(\operatorname{Lift}(A F) \cap G^{\prime}\right)=\pi_{1}^{\prime}\left(i_{1}^{\prime *}\left(\operatorname{Lift}(A F) \cap G^{\prime}\right)\right),
$$

where $G^{\prime}=\mathcal{O}_{p+1+1}\left\{\frac{\partial}{\partial X_{1}}, \ldots, \frac{\partial}{\partial X_{p}}, Z \frac{\partial}{\partial Z}, \frac{\partial}{\partial M}\right\}$ and $\pi_{1}^{\prime}$ is the projection onto the components in $\frac{\partial}{\partial X_{1}}, \ldots, \frac{\partial}{\partial X_{p}}$ and $\frac{\partial}{\partial M}$. 
Now let $\chi=\beta(X) \frac{\partial}{\partial M} \in \pi_{2}\left(i^{*}(\operatorname{Lift}(F))=\pi_{2}\left(i^{*}\left(\pi_{1}^{\prime}\left(i_{1}^{\prime *}\left(\operatorname{Lift}(A F) \cap G^{\prime}\right)\right)\right)\right.\right.$. Notice that the previous equality is amongst ideals in $\mathcal{O}_{p}$, but, alternatively, there is an isomorphism between the two spaces that takes $\beta(X) \frac{\partial}{\partial M}$ to $\beta(X) \frac{\partial}{\partial \Lambda}$. There exists $\xi \in \operatorname{Lift}(A F) \cap G^{\prime}$ such that

$$
\xi=\sum_{i=1}^{p} \xi_{i} \frac{\partial}{\partial X_{i}}+\xi_{p+1} \frac{\partial}{\partial Z}+\left(\beta+\xi_{p+2}\right) \frac{\partial}{\partial M},
$$

where $\xi_{p+1}(X, 0, M)=0$ and $\xi_{p+2}(X, 0,0)=0$. Notice that $\operatorname{dim} \tilde{\tau}(A F)>0$, in fact $\left.\frac{\partial}{\partial Z}\right|_{0} \in \widetilde{\tau}(A F)$ so there exists $\eta \in \operatorname{Lift}(A F)$ such that

$$
\eta=\sum_{i=1}^{p} \eta_{i} \frac{\partial}{\partial X_{i}}+\eta_{p+1} \frac{\partial}{\partial Z}+\eta_{p+2} \frac{\partial}{\partial M},
$$

where $\eta_{p+1}(0) \neq 0$. Without loss of generality we can assume $\eta_{p+1}(0)=1$.

We define $\zeta=\frac{1}{\eta_{p+1}}\left(\eta_{p+2} \xi-\left(\beta+\xi_{p+2}\right) \eta\right)$ and we get

$$
\zeta=\frac{1}{\eta_{p+1}}\left(\sum_{i=1}^{p}\left(\eta_{p+2} \xi_{i}-\left(\beta+\xi_{p+2}\right) \eta_{i}\right) \frac{\partial}{\partial X_{i}}+\left(\xi_{p+1} \eta_{p+2}-\left(\beta+\xi_{p+2}\right) \eta_{p+1}\right) \frac{\partial}{\partial Z}\right) .
$$

We have that $\zeta \in \operatorname{Lift}(A F) \cap G$, so $\chi \in \pi_{2}\left(i^{*}\left(\pi_{1}\left(i_{1}^{*}(\operatorname{Lift}(A F) \cap G)\right)\right)=\pi_{2}\left(i^{*}(\operatorname{Lift}(A f))\right)\right.$, where this equality is amongst ideals of $\mathcal{O}_{p}$.

Now we will prove $\pi_{2}\left(i^{*}(\operatorname{Lift}(A f)) \subseteq \pi_{2}\left(i^{*}(\operatorname{Lift}(F))\right)\right.$. Since $f$ has finite codimension and $\phi$ is quasihomogeneous, by Theorem 4.4 in [6], $A f$ has finite codimension. Let $\chi=$ $\beta(X) \frac{\partial}{\partial Z} \in \pi_{2}\left(i^{*}(\operatorname{Lift}(A f))\right.$, i.e. there exists $\bar{\eta} \in \operatorname{Lift}(A f)=\operatorname{Derlog}(\Delta(A f))$ such that

$$
\bar{\eta}(X, Z)=\sum_{i=1}^{p} \bar{\eta}_{i}(X, Z) \frac{\partial}{\partial X_{i}}+\left(\psi_{p+1}(X, Z)+\beta(X)\right) \frac{\partial}{\partial Z},
$$

where $\psi_{p+1}(X, 0)=0$. By Lemma 3, there exists $\eta \in \operatorname{Derlog}(\Delta(F))$ of type

$$
\eta=\sum_{i=1}^{p} A_{i} \frac{\partial}{\partial X_{i}}+\left(\eta_{p+1}+\beta\right) \frac{\partial}{\partial Z}
$$

where $\eta_{p+1}(X, 0)=\psi_{p+1}(X, 0)=0$, and so $\pi_{2}\left(i^{*}(\eta)\right)=\chi$. The result is proved.

Funding Open Access funding provided thanks to the CRUE-CSIC agreement with Springer Nature

Open Access This article is licensed under a Creative Commons Attribution 4.0 International License, which permits use, sharing, adaptation, distribution and reproduction in any medium or format, as long as you give appropriate credit to the original author(s) and the source, provide a link to the Creative Commons licence, and indicate if changes were made. The images or other third party material in this article are included in the article's Creative Commons licence, unless indicated otherwise in a credit line to the material. If material is not included in the article's Creative Commons licence and your intended use is not permitted by statutory regulation or exceeds the permitted use, you will need to obtain permission directly from the copyright holder. To view a copy of this licence, visit http://creativecommons.org/licenses/by/4.0/.

\section{References}

1. Arnold, V.I.: Wave front evolution and equivariant Morse lemma. Commun. Pure Appl. Math. 29(6), 557-582 (1976) 
2. Casonatto, C., Oset Sinha, R.: A note on the Mond conjecture and crosscap concatenations. J. Singul. 12, 19-26 (2015)

3. Cooper, T., Mond, D., Wik Atique, R.: Vanishing topology of codimension 1 multi-germs over $\mathbb{R}$ and $\mathbb{C}$. Compos. Math. 131(2), 121-160 (2002)

4. Damon, J.: $\mathscr{A}$-equivalence and equivalence of sections of images and discriminants. In: Singularity Theory and Applications (Warwick 1989), Lecture Notes in Math., vol. 1462, pp. 93-121. Springer, New York (1991)

5. Damon, J., Mond, D.: A-codimension and the vanishing topology of discriminants. Invent. Math. 106(2), 217-242 (1991)

6. Houston, K.: Augmentation of singularities of smooth mappings. Int. J. Math. 15(2), 111-124 (2004)

7. Houston, K., Littlestone, D.: Vector fields liftable over corank 1 stable maps. Preprint (2009)

8. Mather, J.N.: Stability of $C^{\infty}$ mappings. II. Infinitesimal stability implies stability. Ann. Math 2(89), 254-291 (1969)

9. Mond, D.: On the classification of germs of maps from $\mathbb{R}^{2}$ to $\mathbb{R}^{3}$. Proc. Lond. Math. Soc. 3(50), 333-369 (1983)

10. Mond, D., Nuño-Ballesteros, J. J.: Singularities of Mappings. The Local Behaviour of Smooth and Complex Analytic Mappings. Grundlehren der mathematischen Wissenschaften, p. 357. Springer. ISBN: 978-3-030-34439-9 (2020)

11. Nishimura, T., Oset Sinha, R., Ruas, M.A.S., Wik Atique, R.: Liftable vector fields over corank one multigerms. Math. Ann. 366(1-2), 573-611 (2016)

12. Oset Sinha, R., Ruas, M.A.S., Wik Atique, R.: Classifying codimension two multigerms. Math. Z. 278(12), 547-573 (2014)

13. Oset Sinha, R., Ruas, M.A.S., Wik Atique, R.: On the simplicity of multigerms. Math. Scand. 119(2), 169-196 (2016)

14. Wall, C.T.C.: Finite determinacy of smooth map-germs. Bull. Lond. Math. Soc. 13(6), 481-539 (1981)

Publisher's Note Springer Nature remains neutral with regard to jurisdictional claims in published maps and institutional affiliations. 